\title{
KRITISCHE ANMERKUNGEN ZU SELBSTHILFE, FREMDHILFE UND PARTIZIPATION IN FREMDBESTIMMTEN ,SELBSTHILFE“- ORGANISATIONEN DER ENTWICKLUNGSPOLITIK
}

\author{
Von J. O. Muller
}

\section{Zum ursprünglichen Konzept der Selbsthilfe}

Es ist die alte, in unserem nordeuropäischen Kulturkreis schon von den frühen Kooperationspionieren gestellte Frage, ob Selbsthilfe nicht ausschließlich von innen und unten, statt von außen und oben her, aus der originären Interessenlage und der Selbstentscheidung der Betroffenen entstehen sollte, wenn sie Bestand haben will und zu einer Bewegung führen soll, die weitgehende politisch-ökonomische Unabhängigkeiten erreicht.

Ein Blick auf den ursprünglichen ethnosoziologischen Ansatz zur Interpretation des Selbsthilfekonzepts macht vorab zwei Erscheinungen manifest, die für Selbsthilfe als konstitutiv angesehen werden dürfen:

1. der auf individuelle und soziale Ziele, Bedürfnisse und Aspirationen bezogene Handlungsantrieb von Personen und Gruppen, den man in der Sozialpsychologie Motivation nennt,

2. die autochthone Bestimmung der Selbsthilfe, als eine ,,am Ort selbst entstandene“, ,,bodenständige“, ,,ortsbürtige“ Handlung - das ist die Wortbedeutung von autochthon.

Historische und zeitgeschichtliche Erfahrungen aus der Kooperationspraxis belegen in der Tat die strategische Bedeutung dieser beiden Erscheinungen für die Entstehung und den Fortbestand von Selbsthilfe-Aktionen, zumindest solange personale und soziale Bedürftigkeit zur Selbsthilfe veranlassen ${ }^{1}$. So dürften diese beiden Erscheinungen auch grundlegend sein für die Gestaltung von ,,Bedingungen“, des ,,Klimas“ “ür die Entstehung von Selbsthilfe, wovon praxiserfahrene und sozial verantwortungsbewußte Entwicklungspolitiker in der Gegenwart zu sprechen begonnen haben, nachdem europäische Genossenschaftskonzepte mehr als 80 Jahre - meistens mit Mißerfolg - nicht europäischen Kulturen zwangsweise akkulturiert worden waren.

Selbsthilfe im ursprünglichen Sinn älterer ethnosoziologischer Forschungsansätze ${ }^{2}$ kann, vereinfacht, auf die folgenden Grundbedingtheiten zurückgeführt werden:

1. bestehende Probleme werden von Personen oder Gruppen als drückend empfunden, lassen einen Zustand der Bedürftigkeit bewußt werden. Es kommt zur Ausprägung konkreter Bedürfnisse, die zur Formulierung von Zielen führen, mit deren Erreichung zugleich die Erfüllung der Bedürfnisse willentlich aktiv angestrebt wird,

2. eine höhere soziale Organisation - z. B. Clan, Stamm, Staat einerseits, Stand, Kaste, Klasse mit organisierter Macht andererseits - also eine Organisation, die sozial und politisch wirksamer organisiert ist als die kleine Gruppe, nimmt sich gewöhnlich Problemen an, die von der kleinen Gruppe nicht selbst gelöșt werden können oder sollen. Selbsthilfe setzt dann den Fall voraus, in dem nicht erwartet wird, daß die höhere Organisation einen befriedigenden Beitrag zur Lösung des selbst als drückend empfundenen Problemkomplexes leisten kann,

1 vgl. J. O. Müller, Voraussetzungen und Verfahrensweisen bei der Errichtung von Genossenschaften in Europa vor 1900. Analyse der Strategien des Genossenschaftsaufbaus in den Frühstadien der industriewirtschaftlichen Entwicklung. Göttingen, 1976.

2 Vgl. insbesondere Steinmetz, S. R., Selbsthilfe, in: Vierkandt, Handwörterbuch der Soziologie, Stuttgart 1931, Neudruck 1959, S. $518-522$. 
3. eine individuelle oder kollektive Initiative für eine Ad-hoc-Aktion wird spontan beschlossen mit dem Streben, den Problemkomplex solidarisch zu lösen. Bei gemeinsam empfundenen Bedürfnissen ist im allgemeinen eine kollektive Aktion zur Zielerreichung zu erwarten.

Ursprünglich ist Selbsthilfe wohl als Handlung in einer Ausnahmesituation entstanden. Ein Handeln im Dissens mit der höheren sozialen Organisation, u. U. sogar gegen die Gebote des traditionellen Rechts, ist nicht ausgeschlossen. Als drückend empfundene Probleme sollen schnell und wirksam gelöst werden. Die selbst empfundene Dringlichkeit drängt zur autonomen Handlung, d. h. einem Handeln auf eigene Initiative und nach eigengesetzlichen Regeln. Wir können darum auch von einem Typus der autonomen Selbsthilfe sprechen, einer Selbsthilfe im eigenen Namen, auf eigene Initiative und mit interner eigengesetzlicher Regelung.

Ein solches, im Verband der größeren sozialen Organisation, unrechtmäßiges Handeln aufgrund einer (sozialen, ökonomischen oder politischen) Notlage kann gruppenintern ethisch legitimiert werden. So steht Selbsthilfe ursprünglich auch in offensichtlicher Loslösung, ja, unter Umständen in Opposition zur Fremdhilfe, sofern diese nicht die selbständige eigenverantwortliche Entscheidung der Bedürftigen über die Verwendung fremder Hilfe zuläßt ${ }^{3}$.

\section{Selbsthilfe und Fremdhilfe im Blick auf die Entwicklungspolitik}

Entwicklungsstrategisch entscheidend für die zentrale Fragestellung der praktischen Entwicklungspolitik im Kooperationsbereich, nämlich einer ,,Anregung der Selbsthilfe“4 zu Zwecken der sozialökonomischen Entwicklung, ist die Erkenntnis des folgenden Sachverhaltes:

1. die Selbstempfindung und Selbstinterpretation von Notlage und Grundbedürfnissen sowie die Selbstformulierung von Aktionszielen zur Bedürfnisbefriedigung, also Selbstund nicht Fremdmotivation,

2. die Entfaltung von Triebkräften zur Selbsthilfeaktion aus Mangelgefühlen, die dem vitalen, personalen und sozialen System entspringen. Die selbstempfundenen Mängel mögen sozialökonomischer und politischer Art sein und mehr oder weniger starke Emotionen wecken,

3. die selbstverantwortliche und dezentrale Organisation kollektiven sozialen Handelns zur Bedürfnisbefriedigung aufgrund entsprechender Motivation,

4. die Entstehung einer derartigen Selbsthilfeorganisation aus autochthonen Sozialverbänden, z. B. Verwandschafts- oder Altersgruppen, Interessengruppen und Schichten, also bereits existenten lokalen Partizipationsgruppen mit hoher sozialer Integration. Es ist vorstellbar, daß daraus eine soziale Verpflichtung zur Teilnahme an der Selbsthilfe entstehen kann, sie mag aber auch freiwillig sein. Andererseits ist damit zu rechnen, daß die autochthone Entstehung von Selbsthilfe sozial restriktiv sein kann; Mitglieder werden dann nur nach bestimmten Auswahlkriterien rekrutiert. Das bedeutet, die SelbsthilfeGruppe kann eine Teilgruppe des größeren lokalen Gruppenverbandes, also Clan, Großfamilie, Gemeinde, Schichtungsstruktur etc. darstellen, d. h. eine „Elite“ mit intern geregelter „Huis-clos-Politik“ des Selbsthilfehandelns sein 5 .

$3 \mathrm{Vgl}$. hierzu die entscheidende Bedingung der Selbstbestimmung über die Verwendung von Fremdhilfe durch die zu Fördernden selbst, bei Krebs, W., Staatshilfe, in: Totomianz, V., Internationales Handwörterbuch des Genossenschaftswesens, Bd. 2, Berlin, 1928, S. $857-859$.

4 Nach dem Begriff von Georg Draheim, Genossenschaften(I), in: Handwörterbuch der Sozialwissenschaften, Bd. 4, Tübingen, Stuttgart, Göttingen, 1965, S. 352.

5 Dafür sprechen z. B. Selbsthilfe-Gruppen im arbeitswirtschaftlichen Bereich oder im Bereich der Durchsetzung von politischen Interessen. 
5. Die selbständige Loslösung von einer höheren sozialen oder politischen Organisation, auch wenn man sich ihr kulturell verpflichtet fühlt mit dem Ziel, bedürfnisbefriedigenden und diesbezüglich zielstrebigen gemeinsamen Handelns.

6. Die Selbstidentifikation mit den notwendig zu lösenden Problemen als Ursprung der teilweise zumindest spontanen Motivation zur Selbstorganisation der Selbsthilfe läßt diese unter einer Art von Sozial- und Rechtsstatut wirken, das man mit ,, dezentraler Autonomie" kennzeichnen darf. Darum die Bezeichnung ,, autonome" Selbsthilfe-Gruppe. Interessant wird jetzt die Frage nach den Möglichkeiten der Entstehung von Selbsthilfegruppen, wenn ihre Einrichtung nicht ,,von innen und unten“, sondern ,,von außen und oben " herrührt, ${ }^{6}$ dabei die Aufgabe der Einrichtung an außerhalb des sozialen Systems stehende lokalkulturfremde Initiatoren, z. B. den Staat und seine einheimischen oder fremden Entwicklungsträger, übertragen wird. Hier ist im Hinblick auf die Entstehung nicht mehr von ,,autochthoner", also ortsbürtiger Entstehung der Selbsthilfe zu sprechen, sondern von ,,allochthoner" d. h. anders-, fremdbürtiger Entstehung. Selbsthilfe erhält dann die Qualität der Fremdhilfe, u. U. auch dann, wenn sie als ,Hilfe zur Selbsthilfe“ oder im Sinne von Draheim als ,,Anregung der Selbsthilfe“ gedacht und beabsichtigt ist.

Hierbei erscheint besonders problematisch, daß die offensichtlich bewährten Wirkungen der autonomen Selbsthilfe bei Trägern der externen, fremdgesteuerten Entwicklungspolitik den Eindruck eines Idealbildes geprägt haben. Vergessen wir aber eines nicht: es sind mehr die Wirkungen als ihre Bedingtheiten, die den - vom Entwicklungspolitiker so genannten Selbsthilfeorganisationen Leitbildcharakter für eine staatliche Entwicklungspolitik verliehen haben. Damit sind wir am neuralgischen Punkt des.Themas.

Ubertragen wir diese Uberlegungen auf die Entwicklungspolitik, so ist immer wieder festzustellen, daß man sich um den autonomen Typus der Selbsthilfe stets weniger gekümmert hat als um den heteronomen Typus, wenn zwar mit dem deutlichen Anspruch, diesen heteronomen in den autonomen Typus umzuwandeln - früher oder später, zunächst nur auf dem Papier, vielleicht aber nie in der Realität.

So sind gerade in der Entstehungsphase der Selbsthilfe-Organisation auch die Ziele der gemeinsamen Aktion im Regelfall heteronom statt autonom und selbstbestimmt; denn ein Dia$\log$ mit den Zielpersonen oder Gruppen findet in aller Regel doch gar nicht statt. Der Planer glaubt i. a. besser als sein Zielpublikum über dessen Grundbedürfnisse befinden zu können. Dabei denkt er im Regelfall einseitig ,,ökonomistisch“, entsprechend der in Industriegesellschaften vorwaltenden ökonomisch-rationalen Sachzwänge. Diesbezügliche Denk- und Verhaltenssysteme sind indessen der autochthonen Gesellschaft selbst bei teilweiser Annahme von modernisierten Arbeits- und Lebensformen fremd. Untersucht man z. B. die Grundbedürfnisse als Handlungsziele des täglichen Lebens, so liegen die Hauptziel-Kategorien zwar zwischen ,,Subsistenz“ (relativ höhere, subjektiv eingeschätzte Bedeutung) und „Einordnung in den monetären und marktwirtschaftlichen Kreislauf“ (relativ geringere, subjektiv eingeschätzte Bedeutung), doch sind dazwischen auch eine Reihe von sogenannten ,,nicht-ökonomischen“ Bedürfnissen mit relativ hoher Priorität im Hinblick auf das tatsächliche Handeln angesiedelt, wie ,Erfüllung von lebenswichtigen, familien- und religionsbezogenen Zielen“, „,Verbesserung der Arbeits- und Lebensbedingungen“ etc. Gerade diese können fallweise größere Posten des verfügbaren familienwirtschaftlichen Budgets langfristig blockieren?

6 Analytische Bezeichnungen, die in der deutschen Geschichte der Kooperation spätestens bei dem Berliner Staatswissenschaftler Victor Aimé Huber auftauchen; vgl. V. A. Huber, Ausgewählte Schriften über Socialreform und Genossenschaftswesen. In freier Bearbeitung hrsg. von Dr. Karl Munding, Berlin, 1894.

7 Analyse nach explorativem Forschungsansatz zur Ermittlung von Handlungszielen von (Zwangs-)Mitgliedern in iranischen Landre- 
Unterstellen wir als Zielgruppen der Selbsthilfe wirtschaftlich schwache Einheiten, also gerade solche, denen Selbsthilfe-Aktionen a priori dienen sollen, so werden in der Entstehungsphase von kooperativen Gruppen vor allem die subsistenzsichernden und -überwindenden Zielkategorien für die Partizipation an Selbsthilfeanregungen motivieren. Vielleicht sind dabei auch jene Zielkategorien zur Erfüllung lebenswichtiger familien- und religionsbezogener Ziele, zur Verbesserung der Arbeitsbedingungen ganz entscheidende Nebenbedingungen für die Motivation zur Teilnahme an Selbsthilfeaktionen - obschon sie für den Planer aus Gründen seiner Konzentration allein auf die in seinem Sinne ökonomisch relevanten Ziele, sicher uninteressant sind. Jedenfalls zeigt sich doch in praxi, daß sie Voraussetzung sein können für mögliches Streben nach marktwirtschaftlichem Engagement der Zielgruppen. Erst an dieser Stelle ergeben sich dann Ziel-Übereinstimmungen zwischen kleinbäuerlichen Produzenten und staatlicher Entwicklungsbehörde. Sind aber die Motivations-Schwellen durch einseitige Orientierung an entwicklungs-ökonomischen Planzielen zu hoch, der Abstand zwischen von unten her gewünschten und von oben her gesetzten Zielen zu groß, so bleibt jede Bereitschaft zur Teilnahme, sofern diese nicht aufgezwungen wird, letztlich illusorisch. Das ist die banale Situation in einer Vielzahl von Selbsthilfeprojekten: Sie sind nicht grundbedürfnis-, sondern planorientiert und erzeugen nicht Partizipation, sondern das Gegenteil: Apathie ${ }^{8}$.

Daraus können wir schließen: In den Händen einer höheren autoritären Planungsbehörde, die möglichst bald sichtbare wirtschaftliche Erfolge nachweisen muß, droht das orginäre Selbsthilfemuster umfunktioniert zu werden: Nämlich zu einem artifiziellen Instrument für staatsgebundene Vorstellungen von Selbsthilfe, Kooperation und Entwicklung. Solche Vorstellungen sind nun einmal anonym von oben nach unten einwirkenden politischen $\mathrm{Pla}$ nungsbehörden zu eigen, zumal wenn sie unverändert Anspruch auf technokratische Modernisierung nach industriekapitalistischem Vorbild erheben. Sie sind im allgemeinen nicht deckungsgleich mit dem Selbstverständnis der Selbsthilfe in den sozialen Zielgruppen solcher Entwicklung. Der Entwicklungsträger kann mit diesem „Behörden-Ansatz“ der „Hilfe zur Selbsthilfe“, allein von oben her, kaum wirksame Entwicklungspolitik betreiben, weil der Inhalt des Begriffs ,Selbsthilfe“ im Verständnis von oben her einerseits, von unten her andererseits gespalten ist.

Die Interpretation von Motiven, Haupt- und Nebenzielen, Grundbedürfnissen als existenznotwendigen Prioritäten der Basisentwicklung durch Entwicklungsträger und $\mathrm{Pla}$ nungsorganismen einerseits, und autochthonen Gruppen andererseits, ist zum Teil widersprüchlich. Widersprüche ergeben sich vor allem aus der Unvereinbarkeit kulturell divergierender Weltbilder und Lebensphilosophien, die dem Mythos hoch spezialisierter, ökonomisierter und technokratischer Entwicklungsvorstellungen verborgen bleiben. Das lehrt jedenfalls vielfältige empirische Felderfahrung. Folglich sind Zielvorstellungen der Behörden für die Entwicklung von unten meistens inkompatibel mit denjenigen der Zielgruppen selbst. Es besteht darum die Gefahr der autoritären Aufoktroierung von Selbsthilfe-Interpretationen durch lokal-kulturfremde Planer und die Gefahr der Degradierung des ursprünglichen Selbsthilfe-Konzepts zum technokratischen Instrument einer unkritischen, weil im Milieu der zu Entwickelnden unüberprüften Úbertragung von Regierungs- und Planungsinteressen von oben nach unten.

formgenossenschaften im Auftrag der F.A.O. der Vereinten Nationen in fünf Dörfern des iranischen Kurdistan, in: Hanel, A., Müller, J. O., On the Evaluation of Rural Cooperation with Reference to Governmental Development Policies-Case Study, Iran. Göttingen. 1976, S. 195-205, und in den Oasen von Kerman, Quelle Fußnote 18, S. 43-54.

8 Dieser Sachverhalt läßt sich leicht aus den nichtveröffentlichten internen Evaluierungen von ,Selbsthilfe“-Projekten staatlicher Entwicklungsträger ableiten. 
Daraus entstehen möglicherweise Probleme einer ethisch ungerechtfertigten, ja illegitim von oben her diktierten Selbsthilfe-Strategie. Das Problem ist um so schwerwiegender, wenn damit wirtschaftliche und politische Interessen hauptsächlich zugunsten des Förderers verknüpft werden, meistens in Gestalt einer kontinuierlichen - aber seit der Kolonialzeit asymmetrisch gebliebenen - ,Do-ut-des-Politik“. Man darf sich nicht wundern, daß bei einer derartigen sozialtechnischen Vergewaltigung eher Apathie als Partizipation das Ergebnis ist. Die Genossenschafts-Geschichte der nordeuropäischen Länder, vor allem Frankreich und Deutschland, aber auch die Geschichte des kolonialen und nachkolonialen Kooperationswesens ist nicht gerade arm an Beispielen dafür.

\section{Selbsthilfe im Blick auf historische Erfahrungen}

Wir verfügen gerade aus den frühen Selbsthilfe-Bewegungen des ,,Kampfes zwischen Arbeit und Kapital" in Europa seit dem zweiten Drittel des neunzehnten Jahrhunderts über kostbare gründungsstrategische Erfahrungen ${ }^{9}$, die zeitlos zu sein scheinen und durchaus eine indikative Bedeutung für die Errichtung von Selbsthilfeorganisationen in Entwicklungsregionen bewahrt haben. Das betrifft ausdrücklich auch die Fremdhilfe. Sie war in der Periode von unten gewachsener, autonomer Selbsthilfe-Institutionen mit Selbstbestimmung und Eigenverantwortung grundsätzlich als Hilfe zur Selbsthilfe intern zugelassen und anerkannt; da nämlich, wo sie der Selbstverantwortung und Selbstentscheidung über Herkunft, Bedingungen und Verwendung solcher Fremdhilfe, von den sozialökonomisch längst funktionstüchtigen Selbsthilfe-Gruppen selbst unterstellt war und selbst kontrolliert werden konnte ${ }^{10}$.

Sechs Kriterien ${ }^{11}$ von existentieller Bedeutung für die Anregung zu sozial-ökonomisch bewährten Selbsthilfe-Organisationen sind aus der historischen Erfahrung abzuleiten. Sie können auch als ,,Bedingungen zur Partizipation an Kooperationsformen “ formuliert werden. Ihre Gültigkeit für Entwicklungsländer heute, wie einst in der Epoche des europäischen Früh- und Hochindustrialismus dürfte schwerlich bestreitbar sein. Sie mögen gerade auch gegenwärtig in der Zeitwende zum Nachindustrialismus, die von einer notwendigen Revision, einer Infragestellung der ,,totalen“ Industrie- und Wachstumswirtschaft mit gesellschaftszerstörender Wirkung geprägt ist, bleibende Bedeutung bewahren ${ }^{\mathbf{1 2}}$.

1. Intellektuelle und funktionale Gründung und Führung von Selbsthilfegruppen durch uneigennützige, ökonomisch unabhängige Promotoren, die von humanitären, religiösen und sozial-politischen Idealen motiviert waren. Sie förderten die Selbsthilfe durch kontinuierlichen Rat und Verhaltensanweisungen im partnerschaftlichen, vertrauten und fachbezogenen Dialog. Sie führten auf strikt ökonomisch orientierter Solidarität basierende strategische Prinzipien der Kooperation ein, die an Basiszielen, sprich: originären Grundbedürfnissen orientiert waren, achteten auf eine schnelle Qualifikation der Partizipanden, damit diese die Selbsthilfeaktion bald selbständig, selbstverantwortlich und selbstverwaltend übernehmen könnten.

9 J. O. Müller, Voraussetzungen . . . a. a. O. und die Vielzahl der dort angegebenen Quellen.

10 Krebs, W., a. a. O.

11 Ergebnis der historischen Analyse von Schriften, insbesondere aus der Feder von primären und sekundären Genossenschaftspromotoren in den europäischen Kernländern, J. O. Müller, Voraussetzungen . . . a. a. O., S. 75-199.

12 Die Zahl der vom Arbeits- und Lebensstil der Industriegesellschaftensich abkehrenden Menschen, die in (meist unpolitischen) kooperativen Gemeinschaften zentral auf den Menschen bezogene Werte leben und diesbezügliche alternative Modelle versuchen, steigt in allen Industriegesellschaften an. Vgl. stellvertretend Walter-Archeion Moritz, die Utopie hat schon begonnen. Die Bedeutung der Landkommunenbewegung für eine humane technologische Gesellschaft. Alpen/Rhld., 1979, und die inzwischen stark angewachsene internationale Literatur zur Gegenkultur. 
2. Diese Selbsthilfe-Förderung konzentrierte sich ausdrücklich auf sozial-ökonomisch homogene Gruppen mit ähnlicher Struktur an Grundbedürfnissen und Existenzzielen, begrenzt in der Gruppengröße, lokal überschaubar, deren Mitglieder ausgewählt wurden im Hinblick auf die wirtschaftliche und moralische Sicherheit für die ökonomische Existenz dieser Gruppen.

3. Gruppengründung und Motivation zur Partizipation basierten auf dem Respekt der individuellen Werte der Persönlichkeit im Kontext der freiwilligen Entscheidung zur Mitgliedschaft und der Selbstformulierung von Aktionszielen entsprechend den lebensnotwendigen materiellen und sozialen Grundbedürfnissen, hier einbegriffen Bildung und Erziehung. Darin lag die Garantie für spontane Kraft, gegenseitiges Vertrauen und soziale Solidarität innerhalb der Selbsthilfegruppe.

4. Wachstum der ökonomischen Solidarität aus der Verpflichtung eines jeden Mitglieds zu regelmäßigen Kapitalbeiträgen und der Mitwirkung an der Betriebsorganisation. Allerdings verblieb dieses gemeinsam zusammengelegte Kapital in den Händen und unter der strengen Verwendungskontrolle der Mitglieder selbst. Später wurde es Grundstock für die Zuleihe an Fremdkapital auf dem privaten und öffentlichen Geldmarkt, zu Anbeginn teilweise unter Selbstauferlegung der unbeschränkten solidarischen Haftung, also einer Haftung der Familien- und Hauswirtschaft mit Bett, Schrank, Herd und Tisch.

5. Aus der Gemeinsamkeit von Notlage und Bedürfnisempfindung selbständiger Zielbestimmung und dem Bewußtsein wirtschaftlicher Gemeinschaft als Quelle sozialökonomischer Stärke entstanden bald auch starke Empfindungen sozialer Solidarität. Ohne sie war die beständige Motivation zur Teilnahme und jene zähe geistige Kraft des Selbstvertrauens undenkbar. Ohne eine derartige soziale Solidarität wäre die Verwirklichung der Selbsthilfe-Utopie unter den gegebenen wirtschafts- und strukturpolitischen Restriktionen einfach unmöglich gewesen.

6. Kontinuierliche kooperative Erziehung, bald als , , learning by doing “, schuf individuelles Verständnis und die Einsicht in den Prozeß der Selbsthilfekooperation. Sie machte den Sinn der selbstauferlegten Verhaltensregeln und ihre Beziehung zur Befriedigung jener selbstformulierten primären Bedürfnisse deutlich. Sie weckte Willen, Disziplin und Treue gegenüber der gemeinsamen Operation. Auch dies konnte wesentlich zur Stimulation von freiwilliger Partizipation beitragen.

Gemessen an diesen Erfahrungen ihres Vorbildes wird leicht einsichtig, warum exogen induzierte Selbsthilfe-Organisationen in Entwicklungsländern bisher auf so schwachen Füßen stehen.

\section{Zum Konzept der Partizipation ${ }^{13}$}

Wir verdanken es vor allem der politologischen Analyse des Begriffs Partizipation, zu erkennen, daß die allein soziologische Interpretation von Partizipation zu entwicklungspolitischen Zwecken nicht ausreicht.

Soziologisch betrachtet, ist Partizipation das regelmäßige Teilnehmen an Interaktionen innerhalb sozialer Gruppen und Gesellschaften. Hierbei wird allein der statische und quantitativ erfaßbare Aspekt formalen Teilnahmeverhaltens wahrgenommen, d. h., die förmliche, u. U. geistig und gefühlsmäßig konforme Ein- und Unterordnung in ein sozialpolitisches Sy-

13 J. O. Müller, Soziale Partizipation - Konzept, Probleme und Bedingungen eines entwicklungsstrategischen Ideals, in: Groeneveld, Meliczed (Hrsg.), Rurale Entwicklung zur Úberwindung von Massenarmut. Hans Wilbrandt zum 75. Geburtstag, Saarbrücken 1978, 57 ff., und die dort zitierten Quellen. 
stem zu einem bestimmten Zeitpunkt. Dies ist vielfach auch die von Herrschaftsträgern erwartete Form der Partizipation einzelner Menschen und Gruppen an den von ihnen festgelegten und kontrollierten Regularien in politisch-sozialen Gebilden. Es fehlen dabei aber Kriterien, die Aussagen über die Art und Weise der Teilnahme, d. h. der kritisch wertenden geistigen und emotionalen Beteiligung berücksichtigen. Hier wird die formale soziologische Interpretation durch die qualitative politologische entscheidend ergänzt, nämlich indem die Frage nach der inhaltlichen Bestimmung der Teilnahme gestellt wird; denn politologisch bedeutet Partizipation das Teilnehmen am politischen Beratungs- und Entscheidungsprozeß in politisch-sozialen Gebilden.

Partizipation kann also soziales, aber ebenso politisches Handeln sein, oder beides zugleich. Letzteres trifft sicher auch für die Entwicklungspraxis zu. Soziale und politische Teilnahme fließen ineinander über, sind darum als zwei Dimensionen der gleichen Aktion anzusehen. Zum Anspruch, lokale und überlokale Entscheidungen mitzutragen und zu verantworten, tritt dann auch die Forderung nach gerechter Teilung des Nutzens aus der Teilnahme am gemeinsamen Handeln. Damit gewinnen sozialökonomische und rechtliche Dimensionen an Bedeutung. So ist zugleich auf den Doppelsinn der Partizipation verwiesen, nicht nur Teilnahme, sondern auch Teilhabe zu sein. Eine wechselseitige komplementäre Beziehung zwischen dem Teilnahmeverhalten und einem daraus für den Partizipanden folgenden Effekt, einem weiterhin verhaltensmotivierenden Nutzen, wird deutlich.

Teilnehmendes Handeln verbindet sich demnach vor allem mit der Erwartung einer Belohnung für dieses Handeln, sei es Belohnung sozialer, ökonomischer, politischer, seelischer oder religiöser Art, je nach dem institutionellen Bereich der Teilnahme.

Nur eine in diesem Sinne ganzheitliche Auffassung der Partizipation auf vielfältige Motivations- oder Zielbereiche gerichtet, ist letztlich entwicklungsrelevant. Nichts anderes kann mit der Formulierung einer Idealvorstellung von „Popular Participation“ durch die Vereinten Nationen gemeint sein ${ }^{14}$, wenn sie zur Realisierung ihrer ,, volkstümlichen“, also durch alle Bevölkerungsschichten breitgestreuten Teilnahme den Einbezug der folgenden drei Typen von Phänomenen nahelegen: 1. aktiver Einbezug der Menschen in den Prozeß der entwicklungsbezogenen Entscheidungsfindung auf allen Ebenen der Gesellschaft, 2. aktiver Beitrag der Menschen zu den Entwicklungsaktivitäten, 3. Beteiligung der Menschen am Nutzen der Entwicklung.

\section{Zur Problematik der Partizipation}

In der Entwicklungspraxis indessen tendieren Regierungs- und Planungsbehörden meistens zu der Eigenart, Partizipation mit Gehorsam gegenüber formalen entwicklungs- und selbsthilferelevanten Verhaltensvorschriften gleichzusetzen. Ihr Verständnis von Partizipation ist auf wenige formale Aspekte, d. h. die angedeutete formale Dimension, begrenzt. Das entspricht den hierarchisch-formalen Rahmenbedingungen, nach denen Projekte gewöhnlich geplant und den Sozialgebilden an der Basis von oben her auferlegt werden. Auferlegt werden bedeutet: ohne die Achtung von vitalen, erfahrenen und kompetenten, somit durchaus faktisch selbstverantwortlichen Sozialsystemen. In diesem Falle werden die Zielgruppen von paternalistischen Entwicklungsagenten schlechthin bevormundet. Polemisch darf man wohl anmerken: letztere hindern sich buchstäblich selbst daran, die wahren Ziel- und Entscheidungsstrukturen, die zur permanenten Partizipation führen könnten, tatsächlich zu entdek-

14. U. N. 1974 Report of the World Social Situation, New York, 1975, S. 255-257. 
ken und in ihrer Projektpolitik zu berücksichtigen. Auf diese Weise gelingt es dann auch nicht, das Vertrauen ihrer Klienten zu gewinnen, d. h. ihren Willen und ihre Bereitschaft zu einer Teilnahmeform im umfassenden geistig-intellektuellen, physischen und emotionalen Verständnis sozialer Partizipation zu mobilisieren. Nur so würde die zur Teilnahme erforderliche Selbstidentifikation mit dem inhaltlichen Bezug und der Zielsetzung der Teilnahme entstehen.

Mit der Anspielung auf einen solchen Idealtypus von Partizipation wird von den Vereinten Nationen ${ }^{15}$ das in der politischen Praxis fast Unmögliche gleich hinzugefügt, nämlich die Notwendigkeit, gesetzliche Barrieren für die Partizipation an entwicklungsfördernden Strategien und Institutionen zu beseitigen - eine politisch derzeit nahezu utopische Forderung.

\section{Bedingungen der Partizipation an Selbsthilfe-Organisationen}

Diese Forderung der UN hat darum eher rhetorischen Charakter; denn für eine verbreitete Motivation zur Partizipation, die verschiedene sozial-ökonomische Schichten der Agrargesellschaft durchdringen will, würde dies die Schaffung einer Reihe von ganz konkreten Voraussetzungen bedeuten:

1. eine menschenwürdige Bodenbesitz- und Arbeitsverfassung (sie ist nach Erfahrungen der bisherigen Landreform-Politik und des dabei effektiv erzielten Erfolges kaum noch ohne die gefürchteten Ansätze revolutionären Charakters zu erreichen ${ }^{16}$ ),

2. die Dezentralisierung von Willensbildung und Entscheidung über Gemeinde- und Gruppenangelegenheiten,

3. ein gesetztes Recht auf formale Partizipation, eine entsprechende Rechtspraxis, die anstrebt, den kritisch und kreativ Denkenden Furcht und Apathie gegenüber Instanzen der staatlichen Hoheit zu nehmen,

4. die Erforschung und Errichtung geeigneter Institutionen, die eine Wahrnehmung dieses Rechts ermöglichen, durch funktionale Bildung und Erziehung, die Anwendung von kulturell angepaßten Sozialisationsformen und spezifischen Lernprozessen zur Teilnahme an dezentralisierten Entscheidungen,

5. die Achtung und Berücksichtigung der autochthonen Zielstrukturen - namentlich in den ökonomisch und politisch weniger privilegierten Schichten - als wichtigste teilnahmemotivierende Triebkraft für das gewünschte entwicklungsrelevante Handeln. Solche Zielstrukturen sind teilweise zumindest identisch mit den Grundbedürfnisstrukturen ${ }^{17}$, von denen so viele reden ohne sich zu bemühen, aus der Selbstdarstellung der Betroffenen heraus zu klären, worin sie in Wirklichkeit eigentlich bestehen und wovon sie im Zusammenhang mit konkreten Zielgruppen reden,

6. der kontinuierliche Dialog zwischen Entwicklungsträger und Zielgruppen auf allen Ebenen des lokalen Entwicklungsprozesses und zwar: Situationsanalyse, Zielformulierung, Wahl der Mittel, Planung, Durchführung und Evaluierung,

7. die glaubwürdige Bereitschaft von Entwicklungsträgern im Falle einer Fremdhilfe zur Selbsthilfe baldmöglichst dezentralisierte Entscheidungen zu respektieren.

Damit sind zunächst Voraussetzungen allgemeiner Art zur Entfaltung der Partizipation am Entwicklungsprozeß genannt, die den Charakter eines übergeordneten politischen Pro-

15. a. a. O.

16. Vgl. hierzu auch die Ergebnisse der 2. Welt-Landreform-Konferenz, Rom, 1979.

17. Zur empirischen Bestimmung von autochthonen Zielstrukturen siehe J. O. Müller, An Empirical Method for the Assessment of Member Goals in Rural Cooperatives. Prerequisite for an Analytical Approach to Evaluation, F.A.O., Rome, 1976 (1978). Zu einer praktischen Anwendung dieser Methode siehe Hanel, A., Müller, J. O., On the Evaluation . . . a. a. O. und dieselben, Improving... vgl. Fußnote 18 . 
gramms annehmen. Sie gelten zweifellos ganz besonders auch für die erwünschte Stimulation der Motivation zur Teilnahme an Selbsthilfeorganisationen. Man wird nicht umhin kommen, sie in geeigneter Weise auf die mikro-soziale Ebene der zu animierenden Selbsthilfegruppen übertragen zu müssen, wenn wirkliche Selbsthilfe - ohnehin zunächst als Entwicklungsprogramm der Fremdhilfe durchaus verdächtig - entstehen soll.

Ohne den Versuch, sich für die politische Realisierung solcher Voraussetzungen wenigstens auf diplomatischem Wege einzusetzen - das gilt insbesondere für landesfremde Entwicklungsträger - wäre der Anspruch, eine Anleitung oder Anregung zur Partizipation geben zu wollen, nicht nur vermessen, er wäre lächerlich. Dies gilt selbst dann, wenn der Staat und seine Behörden dadurch an autoritärer Macht einbüßen würden. Gerade die Furcht vor dem Verlust an politischer Macht ist übrigens die Kernursache für die verbreitete Abwehrhaltung von Regierungen in Entwicklungsländern gegenüber wahren Partizipationsbemühungen.

\section{Empfehlungen zur Gestaltung integraler sozialpolitischer Bedingungen für die Par- tizipation an Selbsthilfe-Organisationen}

Die vorausgegangenen Analysen über Motivation und Teilnahmebereitschaft zu innen- und außenbürtigen Entwicklungsimpulsen, sollten deutlich machen, daß die Fragestellung nach Anleitung, Anregung oder hinführender Erziehung zur Partizipation an Selbsthilfeorganisationen nicht einfach mit einer sozial-technischen Partialstrategie gelöst werden kann. Das gilt zumal im Rahmen der strikten politischen und technischen Gegebenheiten, Anweisungen und Aufgaben eines Entwicklungshilfeprojektes. Denn wir stehen nicht vor einem, wenn auch noch so komplexen technologischen Problem, das meistens mit kontrollierbaren kybernetischen Analysen und hieraus abgeleiteten technischen Prozessen zu lösen ist, sondern wir konfrontieren eine sozio-kulturelle Problematik, die gekennzeichnet ist von einer stets unvollkommenen Transparenz der menschlichen Imponderabilien des Denkens, Wertens und Handelns, zudem in uns fremden, also für uns auch langfristig wenig durchschaubaren Kulturen.

Die Tatsache dieser Begrenzung führt uns zu den folgenden zehn strategischen Empfehlungen im Hinblick auf die Förderung von Selbsthilfegruppen ${ }^{18}$. Sie müssen allerdings im Kontext mit den oben angedeuteten Bedingungen für soziale Partizipation gesehen werden. Unter den gegenwärtig verbreiteten Formen der politischen Realität in Entwicklungsländern haben solche Empfehlungen darum einen eher utopischen Charakter. Aber utopische Gedanken waren in der Sozialgeschichte of t genug ihrerseits Bedingungen und Motive für den Prozeß einer kreativen Veränderung der sozialen Realität in Richtung eines stärker auf den Menschen und seine Grundbedürfnisse orientierten Sozialsystems. Das scheint doch vor allem auch für die gegenwärtige Wirtschafts- und Zivilisationskrise.in den westlichen Industrieländern zuzutreffen. Der nachfolgende Text sei ausdrücklich als ein möglicher Denkansatz unter anderen verstanden, integrale sozialpolitische Bedingungen zur Partizipation an kooperativen Prozessen zu gestalten. Der Begriff der Gestaltung steht dabei bewußt im Gegensatz zum Begriff der Verordnung.

1. Die Verfahrensweise sollte grundsätzlich danach trachten, folgende allgemeine Eigenschaften in allen Förderungsphasen zu wahren:

18 Empfehlungen des Verfassers im Auftrag der Food and Agriculture Organization der Vereinten Nationen, Rom. Ausführlicher Text in: Hanel, A., Müller, J. O., Improving the Methodology of Evaluating the Development or Rural Cooperatives in Developing Countries. Case Study Iran. Food and Agricultural Organization of the United Nations, Rome, 1979 (M/N 0222/EG.79/1/250), S. $59-66$. 
a) dezentralisiert im Sinne der Berücksichtigung existenter physischer und sozialökonomischer Ziel- und Entscheidungsstrukturen,

b) kulturangepaßt im Sinne der Anpassung an die Eigenkultur der betroffenen Ethnien und Gemeinden,

c) bedürfnisorientiert im Sinne der Befriedigung von Primärbedürfnissen, namentlich zunächst zur Stärkung der Subsistenzwirtschaft der zu entwickelnden Individuen und Gruppen als erste Voraussetzung für die darauffolgende regelmäßige Erzeugung eines Mehrproduktes für einen - im Regelfall noch zu organisierenden - Inlandmarkt,

d) entpolitisiert im Sinne der Ausklammerung von staats- und parteipolitischen Zielsetzungen und von politischer Propaganda oder Indoktrination über den Kanal oder das Netzwerk der zu schaffenden Selbsthilfeorganisation,

e) flexibel in der Planung von lokal gezielten Aktionsprogrammen, also unter Verzicht auf fertig konfektionierte Planung durch nationale und außernationale Behörden,

f) koordiniert im Hinblick auf den gleichzeitigen Einsatz von Wissen, finanziellen und materiellen Investitionen zum richtigen Zeitpunkt und durch spezialisierte, interdisziplinär zusammenwirkende Entwicklungsträger, sofern erforderlich; schließlich

g) uneigennützig im Sinne der Bereitschaft aller beteiligten Entwicklungsträger, nicht sich selbst, sondern die Zielgruppe der Förderung als den eigentlichen Entwicklungsapparat anzusehen.

2. Motivation und Partizipation sind abhängig von gegebenen Zielstrukturen, die in sozialökonomischen Schichten und lokalen Subkulturen variieren. Sie sind komplex, über unterschiedliche, aber interdependente, dem kulturellen System eigene soziale Institutionen verteilt und mögen je nach lokaler Situation der Gruppen verschiedene Prioritäten bezüglich ihrer Realisierung, also unterschiedlichen Motivationsdruck besitzen.

Die sozialökonomischen und sozialkulturellen Grundstrukturen der zur Selbsthilfe anzuregenden Sozialsysteme müssen darum zuerst gründlich erforscht, mögliche Vor- und Nachteile für eine Selbsthilfe analysiert werden, die sich z. B. aus heterogenen Sozialstrukturen mit divergierenden Interessen und aus Abwehrhaltungen innerhalb von kulturell identischen System ergeben. Die Ergebnisse dieser Analyse sind konstitutiv für die Entwicklung angepaßter Verfahrensweisen.

3. Bei der Auswahl von Gruppen sollte Überschaubarkeit gewahrt bleiben, besonders geeignete Zielgruppen dürften lokal gewachsene De-facto-Gruppen sein, in denen die Beteiligten gemeinsam ihre Sozialisation und Enkulturation empfangen hatten - unter der Bedingung freilich sozialer Eintracht, die auch in Face-to-face-Gruppen bekanntlich nicht selbstverständlich ist. Kenntnis und Berücksichtigung von Gewohnheiten, Symbolen und religiösen Vorstellungen, ihre Beziehung zu autochthonen Werten in solchen vitalen Gruppen, sind wichtige Voraussetzung für ehrlich gemeinte, gute Wechselbeziehungen mit dem Entwicklungsträger und die Anregung zur Partizipation an Selbsthilfeaktionen.

4. Die Gruppenarbeit konzentriert sich zuerst auf irgendeinen Bereich gemëinsamen Interesses und selbstformulierter Gruppenziele. Es entstehen folglich um Einzelziele, oft Primärbedürfnisse, formulierte Gruppierungen, die dann entsprechend der Befriedigung von Bedürfnissen sukzessive höhere Bedürfniserfüllung anstreben, darunter auch sozialökonomisch und organisatorisch relevante Ziele. Als solche Ziele sind z. B. denkbar: Verbesserung der Nahrungspflanzen und der Tierhaltung, Bodenvorbereitung, verbesserte Anbautechniken, tierische Zugkraft, Flurbereinigung, funktionale Alphabetisierung, Sammlung und Vermarktung von Produkten, biologische Schädlingsbekämpfung, Verbesserung verschiedener Infrastrukturen, wie Straßen, Mikrohydraulik, Einrichtungen der Gesundheitsfürsorge und sanitären Hygiene, Schul- und Fachbildung etc. 
5. Die De-facto-Gruppen sollten unverzüglich angeleitet werden, solche Einzelfunktionen integral und eigenverantwortlich zu übernehmen, um sich mit der Zeit von einer Einzweck-Gruppierung zu einer multifunktionalen Selbsthilfegruppe zu formieren. Dabei sollen sozial akzeptierte, lokal rekrutierte und ausgebildete Träger des Dialogs zwischen unten und oben tätig werden. Es könnte zugleich der Versuch unternommen werden, kooperative Zusammenarbeit und die ständige Diskussion kooperativer Rollen unter Männern und Frauen anzuregen. Der Anreiz zur Erledigung mancher täglicher und jahreszeitlicher Pflichten in gegenseitiger Selbsthilfe, wäre auf Realisierungsmöglichkeiten hin zu überprüfen. Alte, oft vergessene Formen autonomer Zusammenarbeit sind möglichst von Anbeginn wieder zu beleben, allerdings im Rahmen von selbstformulierten Grundbedürfnissen.

6. Die Verwirklichung solcher Ansätze zur Gruppenselbsthilfe wie auch gemeinsames Nachdenken über lokale, unter Umständen kooperative Lösungsmöglichkeiten, sollten evtl. zusammen mit hierzu in möglichst unkomplizierter Weise geschulten Kooperationsagenten durchgeführt werden. Dies würde vor allem die Entwicklung von Motivationshypothesen ermöglichen, mit Hilfe deren dann auch die Entwicklung von spezifischen sozialen Techniken zur Förderung von Partizipation im spezifischen Milieu denkbar und vielleicht auch ohne den Verdacht auf Eigeninteressen der Förderer realisierbar würde.

7. Die Themen für Selbsthilfeinstruktionen sollten von einfach zu bewältigenden Teilstükken oder Unternehmen ausgehen, die sich folgerichtig nach den jahreszeitlichen, ökologischen, ökonomischen und organisatorischen Bedingtheiten leicht zusammenfügen lassen, entsprechend einem Baukastensystem, der behutsamen Entwicklung von unten. Die entsprechende Themenfolge könnte gleichzeitig in leicht verständlicher Weise in den biologischen, technologischen und sozioökonomischen Kontext einer regionalen Entwicklung einbezogen sein, sofern eine solche überhaupt existiert und sich an den lokalen Problemen und Interessen orientiert.

8. Im Verlauf einer solchen Strategie müßte über geeignete Formen der Ausbildung lokaler Animatoren, evtl. Menschen aus vergleichbaren Familien und Betriebsverhältnissen, entsprechend den lokalkulturellen Möglichkeiten nachgedacht werden. Fremde Agenten als Animatoren sollten, sofern möglich, auf ein Mindestmaß reduziert werden. Im Rahmen permanenter Erziehung und Einübung müßten die Mittlerrollen solcher ,Sozialtechniker“ möglichst bald teilweise oder ganz durch repräsentative Mitglieder der Selbsthilfegruppen übernommen werden. Das ist aber nur denkbar, wenn zuerst die selbstformulierten Handlungsziele und dann weitere echte Gruppeninteressen, auch exogen eingeführt, für alle nicht nur glaubhaft, sondern auch nützlich und überzeugend, im Mittelpunkt der Aktion stehen. Als problematisch hat sich allerdings stets eine angemessene Entlohnung für solche Tätigkeiten und die Einordnung solcher Tätigkeiten in formell garantierte, aussichtsreiche Berufswege erwiesen.

9. Eine bedeutende Rolle nimmt die Erziehung ein. Ein Strategem, das freilich aus Gründen der sogenannten mangelnden Rentabilität völlig vernachlässigt worden ist, mit der Ausnahme vielleicht einseitig marktökonomischer Anleitungen, die den kleinen Produzenten häufig nicht vordringlich interessiert haben (bei eigenen Feldstudien in Fiherenana, Madagaskar, hieß es 1966 lakonisch: ,,Man kann das Geld nicht in den Topf stecken und kochen"). Erziehungsprozesse sollten strukturiert sein in Basiserziehung, speziell fachliche Erziehung (einbezogen Selbsthilfe- und Kooperationsthemen) und schließlich lokal erforderliche Ratschläge, evtl. Beratung im Hinblick auf den Kooperations- und Produktionsprozeß, also nicht wie bisher allein auf den letzteren Bereich einseitiger fremdgewollter und meist fremdbestimmter ökonomischer Spezialisierung konzentriert. 
Basiserziehung konzentriert sich auf funktionelle Alphabetisierung (z. B. Kenntnis arabischer Zahlen, einfacher Rechenvorgänge und die Frage, was ist eine Dezimalwaage, wie ist sie gebaut, wo hängen Fälscher ihre verborgenen Gegengewichte an) produktions- und marktfunktionales Rechnen und Buchführen, sanitäre Hygiene und Ernährung etc. Im Laufe solcher Erziehungsmaßnahmen sollte das Bewußtsein für die komplementäre Bedürfnisbefriedigung - sozialökonomisch und politisch rational, wie auch emotional - im Rahmen des ökologischen Bezugssystems geschaffen, wiedergeschaffen oder auch erhalten werden. Spezielle Erziehung bezieht sich auf die erforderlichen soziokulturellen, naturwissenschaftlichen und ökologischen Zusammenhänge, denen das technologische und ökonomische Spezialwissen unterstellt bleiben muß, und nicht umgekehrt, wie in den bisher vergangenen Dekaden des grundsätzlichen Raubbaus durch den internationalen ethnozentrischen Wachstums- und Konsum-Mythos. Die erzieherische Diskussion über entsprechendes Rollenverhalten, biologisch, sozialökonomisch und organisatorisch, gliedert sich an, stets in praktischer Gruppenübung. Immer ist dabei auf die Berücksichtigung lokaler, regionaler und nationaler, also inlandbezogener Bedürfnisse und Ziele zu achten.

10. Sollte es sich in diesem Zusammenhang als notwendig erweisen, Verhaltensregeln im Sinne einer statuarischen Ordnung zu entwickeln, so sollte dabei in den Anfangsphasen keinesfalls der staatliche Gesetzgeber eingreifen. Im Gegenteil sollten alle Möglichkeiten für eine liberale Regulierung der Selbsthilfe oder der anfänglichen Kooperation ausgeschöpft werden, und zwar in Gestalt selbstbestimmter Regularien durch die Mitglieder der Selbsthilfeorganisation selbst. Erst im Zusammenhang später und höher entwickelter Kooperationsbedürfnisse, könnten derartige präkooperative Regulierung mit einer vom nationalen Gesetzgeber vorzuschlagenden Rahmenordnung für Kooperation abgestimmt werden. Dies im Sinne einer möglichen Uberleitung von höherentwickelten Selbsthilfegruppen in eine staatlich garantierte und gesetzlich geschützte Kooperations-Organisation, die dann bei weiterem Wachstum aus der staatlichen Kontrolle zu entlassen ist. Aber dieser Schritt liegt der jungen Selbsthilfeorganisation im allgemeinen noch fern. Nicht zuletzt wegen des mangelnden Vertrauens in den Staat, der grundsätzlichen Skepsis gegenüber jeder anonymen sekundären Organisation, wegen unzureichender formaler Erziehung sowie fehlender grundund kooperationsbezogener Fachbildung. Daran liegt es dann auch, wenn selbst lange bewährte autochthone Selbsthilfegruppen kein Interesse zur ,,Integration “ in größere formelle Kooperationsverbände zeigen und bei ihrer ,Integration“ nach Staatsvorschrift prompt auseinanderbrechen.

\section{Schlußfolgerungen}

Damit dürfte deutlich gemacht worden sein, daß exogene Motivation, Anregung und Anleitung zur Partizipation an Selbsthilfeorganisationen vordringlich eine Strategie der Gestaltung eines breiten Parameters von Handlungsbedingungen voraussetzt. Mögliche Formen eigentlicher Anleitung gründen auf der Erforschung des sozialen Milieus, auf dem partnerschaftlichen Dialog und den daraus zu entwickelnden Formen einer kulturangepaßten Basisund Facherziehung. Probate Konzepte hierzu sind bisher freilich nur in Ausnahmefällen entwickelt worden. Sie harren der wissenschaftlichen und praxisorientierten Erforschung. Ansätze hierzu sind vor allem durch Konzepte der Animation Rurale ${ }^{\mathbf{1 9}}$ und teilweise des Comunity Development entwickelt und bisweilen auch erfolgreich realisiert worden. Wenn

19 Insbesondere nach den soziokulturell und sozialpsychologisch ausgerichteten Methoden des Institut de Recherche et d'Application des Méthodes de Développement, IRAM, Paris. 
sich solche Konzepte in 20 Jahren dennoch nicht durchsetzen konnten, so lag es nicht immer am Grundkonzept an sich, sondern an seiner Einbettung in eine Entwicklungspolitik, die auf sozial-kulturelle Grundstrukturen und lokale Ziel- und Entscheidungssysteme trotz theoretischer Beteuerung letztlich doch nicht hinreichend oder auch überhaupt nicht Rücksicht genommen hat.

Es dürfte ferner deutlich geworden sein, daß sich jeder mögliche Rahmen sozialtechnischer Empfehlungen auf das vitale Aktionsfeld der Gemeinde - möglichst sogar einer Gemeinschaft - und vor allem auf eine großzügige dezentralisierte Organisation bezieht. Viele Beispiele haben doch bewiesen, daß Menschen in sozialen Gruppen des Hinterlandes durchaus zu tauglichen dezentralen Entscheidungen befähigt sind, häufig zu besseren Entscheidungen als dies nationale Behörden, vor allem ihre Entwicklungsplaner, im allgemeinen annehmen. Ohne die Rücksicht auf den hier gespannten Rahmen und seine Interpretation im Kontext der Lokalkultur, ist mit gewisser Sicherheit jede strategische Mühe um Anreize für soziale und wirtschaftliche Partizipation letztlich zum Scheitern verurteilt.

So liegt es schließlich auch nahe, daß unter den gegenwärtigen Bedingungen der nationalen und internationalen Entwicklungspolitik die Entstehung von selbsthilfeorientierten Kooperationsformen unter fremder also nicht autonomer Initiative recht pessimistisch zu beurteilen ist. 


\title{
Critical annotations on self-help, foreign help and participation in extraneously determined self-help organization of development policy.
}

\author{
By Julius O. Muller
}

The effects of autonomous self-help, which socioeconomically have proved true in history, appear to the agents of an externally controlled development policy to offer a model for the development goals of the state policy in underdeveloped countries. But, thereby, the internal legitimacy of this model as condition for participation and the desired effect on development have, until now, been left out of consideration. On the basis of the ethnosociological historical approach, two phenomena are presented which can be considered as constituting the original concept of autonomous self-help: 1) the motivation of people and groups with reference to self-perceived and self-formulated, individual, and social goals (basis) needs and aspirations, and thus 2) the autochthonous determination of self-help as an action originating from the locality itself and from the self-perception of a need. This concept is exempt from foreign intentions concerning self-help. It is in contradiction with foreign help insofar as foreign help does not allow the autonomous and free decision of the needy people as to the utilization of foreign help.

With regard to the development policy and its socioeconomic strategy for promoting selfhelp, the type of autonomous self-help is compared to the type of foreign, externally induced self-help intention of a foreign agent of development. The first typeoriginatesfrom a polyvalent, practical, because as a whole psycho-physically perceived, goal parameter and is consequently based on strong motivation. The second type is rather founded upon one-sided economic and materialistic objectives which are usual for the technocratic agents of development. It is more dissociated from the realities of life and from the basic needs. For that reason, it seems that the needy are less motivated to accept it. The incongruence and incompatibility of the goal parameter of these two types of self-help explain the frequently observed apathy which target group show towards development policy measures imposed from above and from outside.

The claim of transforming, sooner or later, thy type of heteronomous self-help into the autonomous type by deofficialization cannot be realized because the original concept of autonomous self-help is disregarded - or feared by the agents of state development because of its decentralized autonomy. There is a danger that 1) planning officials who are not familiar with the local culture authoritatively dictate their interpretations of self-help; and 2) that the original self-help concept degenerated into the technocratic instrument of an uncritical - because it has not been tested among those to be developed - downward transfer of the government's and the planners' interests. The problem becomes even more crucial if it involves the economic and political interests of the agents of development - very of ten in the form of a „,development policy“ which has remained asymmetrical since the colonial era and which frustrates a priori the confidence of the masses regarding their participation in the government's development programmes.

The sociological condititons for participation are dealt with in detail. Recommendations are made for creating - and not instituting - sociopolitical conditions for participation in selfhelp organizations. The author worked them out for the Food and Angriculturale Organization of the United Nations. 